\title{
自组装策略调控表面反应
}

\author{
刘忠范 \\ (北京大学化学与分子工程学院, 北京 100871)
}

\section{Steering On-Surface Reactions with Self-Assembly Strategy}

\author{
LIU Zhong-Fan \\ (College of Chemistry and Molecular Engineering, Peking University, Beijing 100871, P. R. China)
}

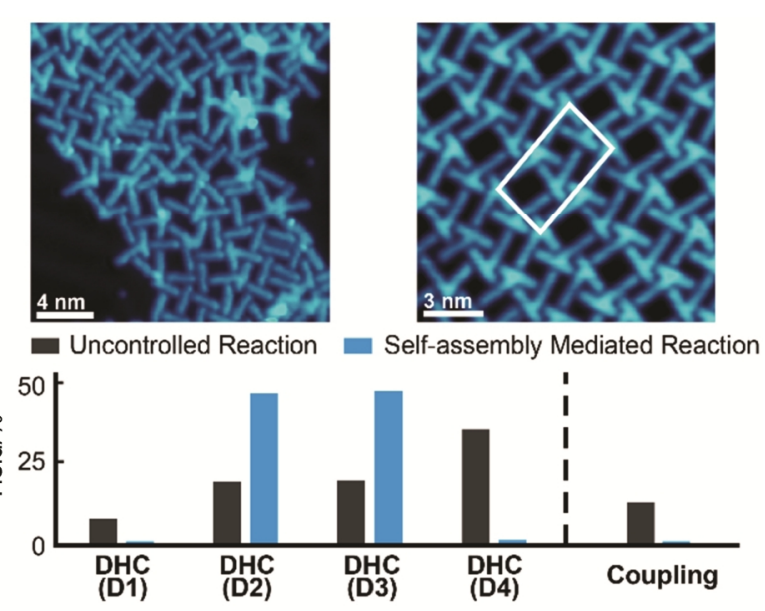

表面反应，即在固体表面发生的化学反应，一 直是化学学科的研究焦点。近年来, 人们借助扫 描隧道显微镜(STM)等表面分析技术, 在表面上成 功实现大量的有机反应如 Ullmann 偶联 ${ }^{1}$ 、Glaser 偶联 ${ }^{2}$ 和 Bergman 环化 ${ }^{3}$ 等。

表面反应涉及到固体表面结构和反应分子本 征性质, 就像一个硬币的两个方面。固体表面具 有催化活性的增原子、表面平台和台阶、表面限 域和晶格诱导效应等 ${ }^{4-7}$ 均可对表面反应进行有效 调控, 这实际上是调变了 Arrhenius 公式中的反应 能垒。然而, 人们常忽视分子自身的排列方式和 相互作用对表面反应的影响, 即调变 Arrhenius 公 式中的指前因子。后者的相关研究在文献中鲜见 报道。

最近北京大学吴凯教授课题组发现, 分子自 组装可以精确调控表面反应, 相关结果发表在

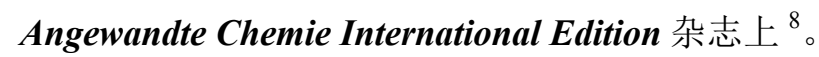
利用 STM 技术, 他们研究了 $\mathrm{Au}(111)$ 表面上 4,4'二(2,6-双氟代吡啶-4-基)-1,1': $4^{\prime}, 1^{\prime \prime}$-三联苯分子的 自组装及其对表面反应的调控。研究发现, 当表 面升温至 $520 \mathrm{~K}$ 以上时, 分子会发生两个通道的 反应, 即脱氢环化反应和脱氟偶联反应。脱氢环 化反应产物共有 4 种同分异构体, 每种同分异构 体又包含 2 种表面手性异构体; 脱氟偶联反应则 包含 2 种表面构象异构体。快速升温造成分子在 表面混乱排列时, 两个反应通道的所有 10 种可能 反应产物都被实验观测到。若缓慢升温, 分子在 反应前先自组装成有序结构, 更高温度反应时只 形成脱氢环化反应通道的 2 种产物, 而脱氟偶联 反应通道被彻底切断。通过监控分子自组装结构 在反应过程中的演变, 他们确认处于自组装体中 的反应物分子原位发生反应。反应分子的自组装 
导致了产物分布的窄化和反应选择性的提高。这 一研究不仅开启了分子自组装调控表面反应的先 河, 更为表面反应的调控提供了新思路。该研究 是与中国科学技术大学邵翔教授, 美国普渡大学 廖佩琳教授和丹麦奥胡斯大学 iNano 中心 Kurt Gothelf 教授合作完成的。

\section{References}

(1) Cai, J.; Ruffieux, P.; Jaafar, R.; Bieri, M.; Braun, T.; Blankenburg, S.; Muoth, M.; Seitsonen, A. P.; Saleh, M.; Feng, X.; Mullen, K.; Fasel, R. Nature 2010, 466, 470. doi: 10.1038/nature09211

(2) Liu, J.; Chen, Q.; Xiao, L.; Shang, J.; Zhou, X.; Zhang, Y.; Wang, Y.; Shao, X.; Li, J.; Chen, W.; Xu, G. Q.; Tang, H.; Zhao, D.; Wu, K. ACS Nano 2015, 9, 6305. doi: 10.1021/acsnano.5b01803

(3) Sun, Q.; Zhang, C.; Li, Z.; Kong, H.; Tan, Q.; Hu, A.; Xu, W. J. Am.
Chem. Soc. 2013, 135, 8448. doi: 10.1021/ja404039t

(4) Björk, J.; Hanke, F.; Stafström, S. J. Am. Chem. Soc. 2013, 135, 5768. doi: $10.1021 / \mathrm{ja} 400304 \mathrm{~b}$

(5) Bieri, M.; Nguyen, M. T.; Gröning, O.; Cai, J.; Treier, M.; Aït-Mansour, K.; Ruffieux, P.; Pignedoli, C. A.; Passerone, D.; Kastler, M.; Müllen, K.; Fasel, R. J. Am. Chem. Soc. 2010, 132, 16669. doi: $10.1021 /$ ja107947z

(6) Adisoejoso, J.; Lin, T.; Shang, X. S.; Shi, K. J.; Gupta, A.; Liu, P. N.; Lin, N. Chem. Euro. J. 2014, 20, 4111. doi: 10.1002/chem.201304443

(7) Lipton-Duffin, J. A.; Ivasenko, O.; Perepichka, D. F.; Rosei, F. Small 2009, 5, 592. doi: 10.1002/smll.200801943

(8) Chen, Q. W.; Cramer, J. R.; Liu, J.; Jin, X.; Liao, P. L.; Shao, X.; Gothelf, K. V.; Wu, K. Angew. Chem. Int. Ed. 2017, doi: 10.1002/anie. 201700745 